\title{
Method for Calibrating a Standard Volt Box
}

\author{
Bernadine L. Dunfee
}

(August 22, 1962)

\begin{abstract}
CA volt box provides several discrete ratios and permits the accurate measurement of direct voltages (maximum about 1,500 volts) through their reduction by suitable factors to values within the measuring capability of a potentiometer. The ratios of a volt box are measured against those of a standard using a "difference" technique. The standard volt box used at the National Bureau of Standards possesses those design features required for a ratio standard and lends itself to a "self-calibration" technique. This paper describes a method for measuring its ratios at rated and above rated voltage to an accuracy of 10 parts per million or better using a Direct Reading Ratio Set and a group of resistance standards. Although the method is described in its particular application to the NBS standard it can be used equally well in the measurement of other ratio networks. Errors that might appear in the measurements and procedures for evaluating their magnitudes are outlined and the derivation of a general expression that defines all ratios in terms of measured quantities is presented in an appendix. Comments and further details regarding the standard and its use are included.
\end{abstract}

\section{Introduction}

The value of a measured voltage, either direct or alternating, is derived from the national standard of emf. At the National Bureau of Standards the unit of voltage is maintained in terms of the mean value of emf of 48 saturated cells. This emf is only slightly larger than one volt so that the accurate measurement of larger voltages of any frequency usually requires the design of special ratio networks that can serve as voltage ratio standards, because of their stability and high accuracy. The volt box and the potentiometer with which it is used are classic examples in the measurement of moderately high direct voltages.

The volt box referred to here, as differentiated from other types of $\mathrm{d}$-c ratio devices (or voltage dividers), provides several discrete ratios so that the voltage to be measured (maximum about 1,500 $\mathrm{v}$ ) is reduced by a suitable factor to a value within the measuring capability of a potentiometer. Insertion of a volt box in a measuring circuit introduces an error in the measurement unless the volt box divides perfectly or the corrections to its nominal ratios are known and applied. In any event, each ratio must be measured, preferably at rated voltage and at a reduced voltage to evaluate the effects of self heating and leakage resistance.

The method of calibration employed at NBS - utilizes a difference technique in which the potential at each binding post of a volt box under test is measured with respect to that at the corresponding binding post of the standard volt box, with both instruments connected in parallel and for the same nominal ratio. Special features must therefore be incorporated in the standard volt box to assure long term stability and the resistance sections must be arranged in a proper sequence with respect to magnitude so that a "self-calibration" procedure is possible and accurate to better than 10 parts per million (ppm). The standard volt box used at NBS, as well as a method developed for its calibration, have been described by Silsbee and Gross [1]. ${ }^{1}$

A second method for calibrating the standard volt box was recently developed and has been under observation for more than a year. The associated circuitry and components have been incorporated in a semiportable test console and the only connections that need be made at the time of test are those to the d-c supply and to the volt box. The present paper describes this method and uses the NBS standard volt box to illustrate the technique. Additional comments are included on the use of the standard volt box in calibrating other volt boxes.

\section{Comments on the Calibration of a Volt Box Against the Standard}

A volt box consists, in general, of a large number of resistance coils connected in series. At both the low- and high-potential ends and at appropriate junctions along the resistor, taps are brought out to binding posts to form the high voltage side. The maximum voltage that can be applied between the zero post and any of the others is governed by the design of the resistance coils and is so marked at each binding post. In addition, the resistance of the lowest section is tapped at an appropriate point and

\footnotetext{
${ }^{1}$ Figures in brackets indicate the literature references at the end of this paper.
} 
leads are brought out from the zero potential end and this tap point to binding posts, forming the low voltage side for connection to a potentiometer. Any given voltage ratio is defined as the ratio of the voltage applied to the high side to that appearing across the low side. These ratios are integers and a given volt box, for example, may cover values from $1,500 / 1.5$ to $3 / 1.5$ if it is to be used with a $1.5-\mathrm{V}$ potentiometer or values from $1,500 / 0.15$ to $3 / 0.15$ for a $150-\mathrm{mv}$ potentiometer.

The test instrument is calibrated with reference to the standard in the circuit shown schematically in figure 1. The two volt boxes, having the same nominal ratio, are connected in parallel through leads $l_{1}$ and $l_{2}$ across a suitable d-c source. The measuring branch, consisting of a detector, a low range potentiometer, and a reversing switch (not shown), is inserted, in voltage opposition, into gaps identified at positions $v_{1}, v_{2}, v_{3}$, and $v_{4}$. For each position the output of the potentiometer is adjusted for a null on the detector so that at balance the potential difference between corresponding points on the two volt boxes is equal to that of the potentiometer. The potential differences $v_{1}$ and $v_{4}$ are small since they are essentially the potential drops in leads $l_{1}$ and $l_{2}$; similarly, if the volt box under test is of high quality, potential differences $v_{2}$ and $v_{3}$ will be small. Thus only moderate accuracy is required in the measurement of the potential differences in order to realize an extremely high accuracy for the values of ratio.

The equation that defines the ratio of the volt box under test in terms of the ratio of the standard and the measured potential differences may be derived as follows. Let the respective polarities be as shown in figure 1. By definition, the true ratio of the volt box under test is

$$
N_{x t}=N_{x n}\left[1+\mu_{x}\right]=\frac{V}{V_{a b}}
$$

where $\mu_{x}$ is the correction in proportional parts and $N_{x n}$ is the nominal ratio.

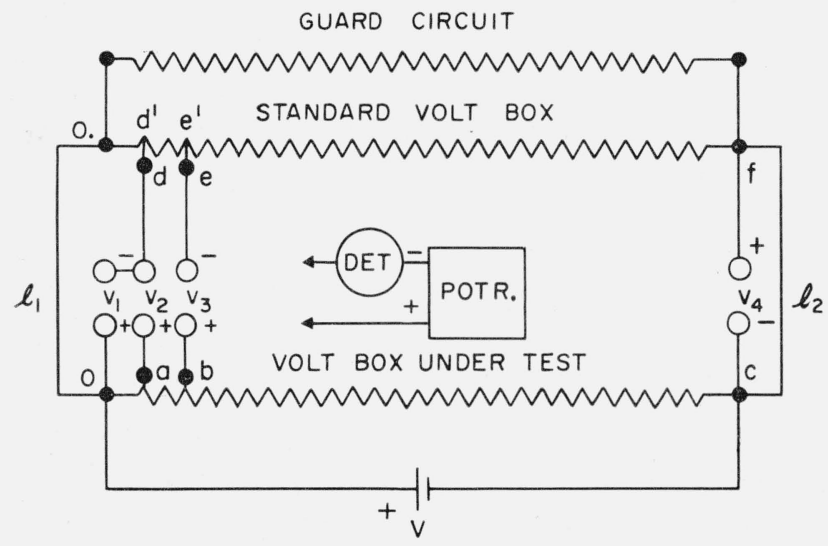

FIgURE 1. Circuit for calibrating a volt box.
Summing the potential drops around loop "badeb" gives

or

$$
V_{a b}-v_{2}-V_{d e}+v_{3}=0
$$

$$
V_{a b}=V_{d e}+\left(v_{2}-v_{3}\right) \text {. }
$$

By definition, the true ratio of the standard volt box is

or

$$
N_{s t}=N_{s n}\left[1+\mu_{s}\right]=\frac{V_{d f}}{V_{d e}}
$$

$$
V_{d e}=\frac{V_{d f}}{N_{s n}\left[1+\mu_{s}\right]}
$$

where $N_{s n}$ is the nominal ratio of the standard and $\mu_{s}$ is the correction in proportional parts. Substituting this value in eq (2) gives

$$
V_{a b}=\frac{V_{d f}}{N_{s n}\left[1+\mu_{s}\right]}+\left(v_{2}-v_{3}\right) \text {. }
$$

Summing the potential drops around loop "defcod" we have

or

$$
-V_{d f}-v_{4}+V-v_{1}=0
$$

$$
V_{d f}=V-\left(v_{1}+v_{4}\right) \text {. }
$$

When this expression is substituted in eq (3) $V_{a b}$ becomes

$$
V_{a b}=\frac{V-\left(v_{1}+v_{4}\right)}{N_{s n}\left[1+\mu_{s}\right]}+\left(v_{2}-v_{3}\right)
$$

and if substituted in eq (1) the true ratio of the volt box under test becomes

$$
N_{x n}\left[1+\mu_{x}\right]=\frac{V N_{s n}\left[1+\mu_{s}\right]}{V-\left(v_{1}+v_{4}\right)+N_{s n}\left[1+\mu_{s}\right]\left(v_{2}-v_{3}\right)} .
$$

On rearranging terms and remembering that $N_{x n}=N_{s n} \approx N_{s n}\left[1+\mu_{s}\right]$ the above expression becomes

$$
\left[1+\mu_{x}\right]=\frac{\left[1+\mu_{s}\right]}{1-\frac{\left(v_{1}+v_{4}\right)}{V}+N_{s n} \frac{\left(v_{2}-v_{3}\right)}{V}}
$$

so that

$\mu_{x}=\mu_{s}+\frac{\left(v_{1}+v_{4}\right)}{V}-\frac{\left(v_{2}-v_{3}\right)}{V / N_{s n}}$ plus higher order terms.

If $v_{1}, v_{2}, v_{3}, v_{4}$, and $V$ are expressed in volts then, neglecting higher order terms, the correction to the nominal ratio is

$\mu_{x}=\mu_{s}+\frac{\left(v_{1}+v_{4}\right)}{V}+\frac{\left(v_{3}-v_{2}\right)}{V / N_{s n}}$ in proportional parts.

If $v_{1}, v_{2}, v_{3}$, and $v_{4}$ are expressed in microvolts and $\mu_{s}$ in $\mathrm{ppm}$ the correction is given in ppm. It should be noted that if the relative polarities of the supply voltage $(V)$ and the potentiometer differ from those assumed in figure 1, the appropriate sign must be given to the recorded values of the measured quantities. 
As will be seen later in the discussion on the calibration of the standard volt box, the measured resistances of all sections and their summations are referred to the resistance of the first section which is chosen to lie between junctions $d^{\prime}-e^{\prime}$. Thus the true ratio of the standard volt box, given as $\frac{V_{d f}}{V_{d e}}$ in the above derivation, is consistent with the measured ratio. (The effects of rod resistances, ${ }^{2}$ such as o.d.', on the measured ratios are discussed in the section on errors.)

It is advisable that the potential drops in leads $l_{1}$ and $l_{2}$ be small in order to achieve the highest accuracy in the measured ratios without placing undue demands on the accuracy required in the measurement of the potential differences. A slight modification made in the test circuit would eliminate the lead effect and at the same time simplify the computation of test data. Referring to figure 2, let small resistances $R_{1}$ and $R_{2}$ be connected in the $l_{1}$ and $l_{2}$ branches with the d-c supply connected between their sliding contacts. The potential drops in the two sections of branch $l_{1}$, as well as those in branch $l_{2}$, are in opposition so that it is only necessary to adjust the respective sliders to achieve cancellation, which is indicated by a null on the detector connected at the $v_{1}$ and $v_{4}$ positions, respectively (see fig. 1). For this condition, the second term on the right of eq (5) becomes zero.

\section{Standard Volt Box}

A volt box must have several special features incorporated in its design if it is to serve as a standard in making ratio measurements to an accuracy of a few ppm. (1) Some provision must be made for reducing the effects from leakage paths to negligible amounts; (2) the selection and arrangement of the resistance wire must guarantee freedom from the effects of relative humidity as well as those arising from self-heating; (3) the corrections to the ratios should be small and the resistances of the tapped sections proportioned so that a "self-calibration" procedure of sufficient accuracy is applicable.

\subsection{Leakage}

The volt box is a high resistance network operating at rather high voltages so that careful consideration must be given to minimizing leakage paths into which a portion of the current may be diverted from points along the working resistance. Effects from such parallel paths may be reduced sufficiently by keeping the insulation resistance high. For example, if the total resistance of the high side is $250,000 \mathrm{ohms}$, the resistance of a leakage path bridging this section must be $2.5 \times 10^{9} \mathrm{ohms}$ for an uncertainty no greater than 0.01 percent, and $2.5 \times 10^{11} \mathrm{ohms}$ if an error no greater than $1 \mathrm{ppm}$ is required. This protection

2 The rod resistances mentioned throughout the text refer to the leads which extend from the resistance sections to the binding posts where connections are made to the external circuit.

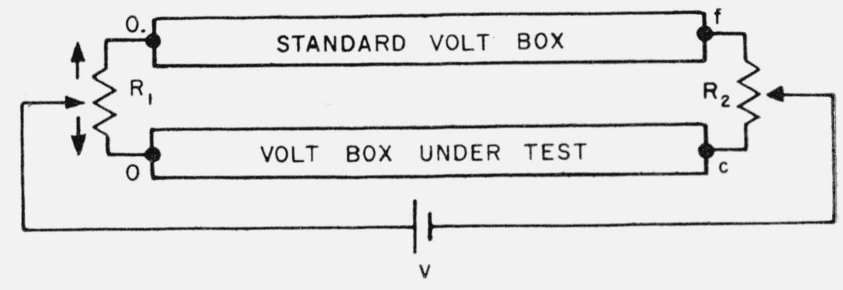

FiguRE 2. Branches for compensating lead drops.

may not be adequate in maintaining long-time stability because of possible accumulation of moisture and contamination across the insulating surface or possible deterioration of the insulation over long use. Additional protection must be provided by a guard network which, in effect, maintains the shields surrounding the resistance sections at the same potential as that of the resistance section within.

The standard volt box discussed here is equipped with a guarding resistance whose corresponding sections can be connected in parallel with the working circuit when the $15-\mathrm{v}$ and higher ranges are used. Metal guard rings surrounding each binding post of the working resistance and connected to the corresponding binding post of the guarding resistance prevent leakage across and through the top panel. The working resistance is divided into sections of not more than $25,000 \mathrm{ohms}$ with the resistance coils of each section mounted on separate insulating panels. Each of these insulating panels is fastened to the top panel through a metal block that is connected to the guard resistance and maintained at the appropriate potential to prevent leakage from one insulating panel to another. In use, leads that connect the standard volt box to the volt box under test and to the other parts of the measuring circuit carry their own shields which are maintained at their appropriate potentials through connections to the binding posts of the guard circuit.

\subsection{Humidity and Self-Heating}

Any effect from relative humidity on a properly guarded volt box originates at the resistance coils. It has long been known that certain insulating materials used for wire covering and for protective coatings of resistance coils are susceptible to changes in relative humidity and produce changes in the resistance of the coils. Although the effect might be expected to be negligible in materials available today or, if not, to be sufficiently suppressed in ratio devices, the matter must be considered if accuracies of a few ppm are desired. The particular standard volt box referred to in the original paper was found to have seasonal variations as large as $30 \mathrm{ppm}$ as a result of changing humidity, so that provision was made for continuously supplying dry air at a pressure slightly higher than atmospheric. In 1960 a new unit was purchased in which the individual resistance coils are hermetically sealed. To date, no measurable seasonal variations because of changing humidity have been observed. 
In theory, the effects from self-heating could be measured and the appropriate correction applied; but these effects can be avoided by designing the unit for lower rated current, by selecting the proper kind of resistance material and by providing the coils with sufficient cooling surface within an adequate exchange volume. Both the original standard and the one recently acquired have a working resistance of $3331 / 3 \mathrm{ohms} / \mathrm{v}$ and each coil is wound with a single layer of manganin wire taken from the same spool. There is sufficient freedom from self-heating that, with double rated voltage applied, the correction differs from that at reduced voltage by less than 5 ppm.

\subsection{Self-Calibration Feature}

The most difficult and critical problem encountered with any standard is the determination of its corrections to an accuracy considerably better than that required for the test instrument. The method should not only satisfy the immediate requirements in accuracy but should be capable of providing still better accuracy in the anticipation of future demands.

One of the most powerful measuring techniques available and one capable of the highest accuracy is that in which small differences are measured between like and nearly equal quantities. The circuit suggested by Silsbee and used in the designs of both standard volt boxes lends itself to this "difference" technique. Its arrangement and adaptation to difference measurements can be best understood by referring to figure 3 . The circuit has 21 tapped sections beginning with the " 0 to 0.15 " section of 50 ohms and continuing to the "750 to 1500 " section of 250,000 ohms. $^{3}$ This resistance chain can be considered as being formed of four groups $M_{1}, M_{2}, M_{3}$, and $M_{4}$ in which group $M_{1}$ serves as the first section of group $M_{2}, M_{2}$ as the first section of group $M_{3}$, and $M_{3}$ as the first section of group $M_{4}$. Being so considered the following pattern is observed. Each

3 The last section of $250,000 \mathrm{ohms}$ is not present in the standard volt boxes at NBS. It is included here for completeness. group contains six sections. The first five sections of any group have nominally equal resistances while the sixth section has a value nominally equal to the sum of the first five. It is possible, therefore, to measure the small differences between the first 50ohm section and each of the remaining four sections of group $M_{1}$, and follow this by measuring the difference between the 250-ohm section and the sum of the first five sections. Since $M_{n-1}$ forms the first section of $M_{n}$ this measuring sequence can be repeated for each group in turn. Because of the continuity within a group and from one group to the next, the resistance of any portion summed from the zero end, compared with that of the first 50-ohm section can be computed from the nominal value of their ratio and the sum of the measured differences.

\section{Direct Reading Ratio Set Test Method}

With the rapid growth of standards laboratories evident in recent years, voltage ratio standards of the type discussed here are no longer the exclusive property of the National standardizing laboratory. The method recently adopted at NBS for calibrating the standard volt box and described herein makes use of equipment that is readily available in many of these laboratories. Thus, from the information that is given on both the theoretical and practical aspects of the method, the standards laboratory can periorm the calibration without recourse to NBS. The method is not limited solely to those volt boxes having the particular sequence of sections indicated in figure 3 but can be used for any network in which the resistance of each section is nominally equal to the resistance of some combination of lower-valued sections. The only change lies in the defining equation and its derivation.

\subsection{General Comments}

A Wheatstone bridge network is used in which the successive difference measurements, referred to in the previous section, are made by inserting in turn each section or summation of sections in the unknown arm of the bridge. Aside from a d-c supply and detector, the only equipment required is a Direct

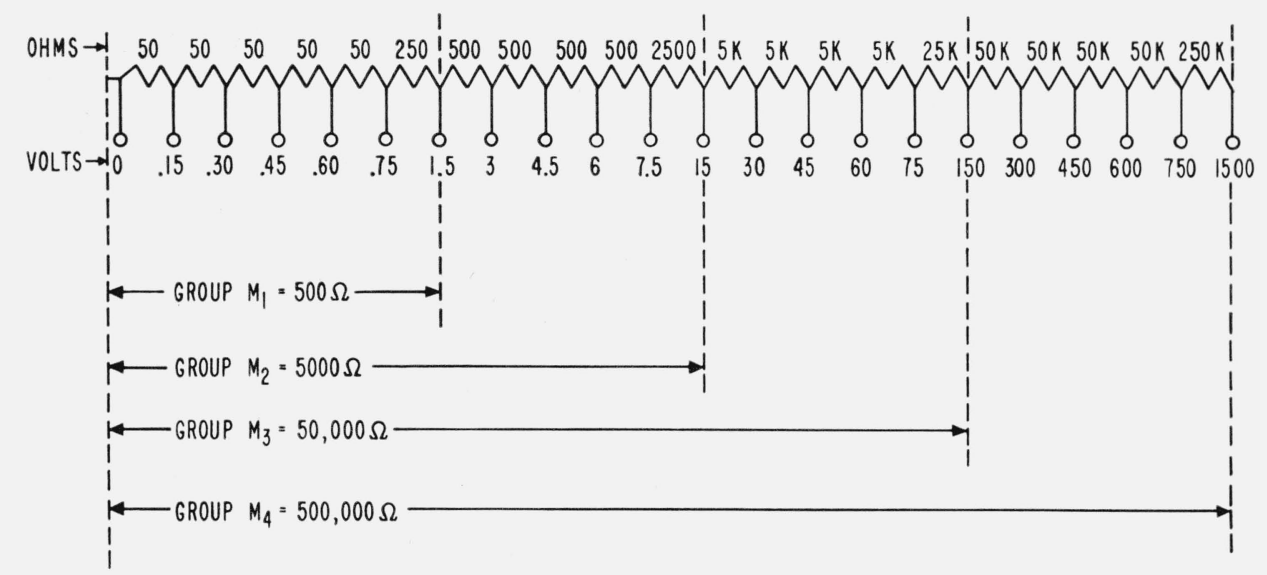

FiguRe 3. "Self-calibrating" network of a standard volt box. 
Reading Ratio Set (DRRS) and a group of seven resistance standards: one each having the values 50 , $200,500,2,000$, and 5,000 ohms, and two having a value of $50,000 \mathrm{ohms}$. (Other types of resistors or resistance assemblies could be substituted for the standard resistors if they remain stable during the sequence of measurements.) The execution of the test is quite rapid and with the circuit incorporated in a suitable console a complete calibration including computation can be made in an hour by experienced personnel.

In the original method the test voltage was limited to some extent because of possible heating of the components. For example, measurements on the $50,000-\mathrm{ohm}$ sections, if made at their rated voltage of $150 \mathrm{v}$, required a power dissipation of $2.25 \mathrm{w}$ in one of the standard resistors. Since it is advisable to keep the power dissipation appreciably less than this amount, periodic calibrations were made at reduced voltage after it was initially established that changes arising from self-heating were negligible. The DRRS method has the advantage that all sections can be measured at their rated voltage. Under this condition the maximum power dissipation required of the standard resistor is $0.45 \mathrm{w}$. With some of the resistor assemblies now available the method can be extended to measurements at 200 percent rated voltage so that the overload capability of the standard volt box can be easily determined.

The method has a further advantage in that the positions of the supply and galvanometer (detector) relative to the circuit are such that the change in circuit resistance as seen by the galvanometer is quite small. Thus the damping and galvanometer response are little affected throughout the measurements.

\subsection{Calibration Procedure}

The measuring circuits are indicated schematically in figures $4 \mathrm{a}$ and $4 \mathrm{~b}$; the reversing switch for the supply is omitted to preserve simplicity. Resistances $A$ and $B$ represent the two arms of a DRRS (including leads) each having a nominal value of 100 ohms and $S$ is a standard resistor always serving as a "Dummy" resistance and having a nominal value equal to that of the volt box section being measured. The guard branch that appears in figure $4 \mathrm{~b}$ includes a resistance of about $80 \mathrm{ohms}^{4}$ in series with the appropriate guard section of the volt box; and the leads of the measuring circuit are of such length and size that at balance the DRRS reads near midscale.

Measurements on the first five sections are made with the circuit of figure $4 \mathrm{a}$. The d-c supply is connected to the 1.5 binding post and the detector to the zero dot $(0$.$) so that rod resistances l_{1}, l_{2} \ldots$ $l_{n-1}$ appear in the $A$ - and $S$-arms of the bridge rather than as part of the resistance being measured. Inequalities among the rod resistances can introduce errors in the measured ratios and are of greater significance when the low-valued sections are measured. There are three alternatives for locating the rod resistances: (1) both rods may be connected as part of the section being measured, (2) one rod may be so connected and the other located in the $S$-arm, and (3) they may be arranged as shown in figure $4 \mathrm{a}$. The relative magnitudes of the rod resistances in the NBS standard volt box indicate figure $4 \mathrm{a}$ as the preferred connection. ${ }^{5}$

The remaining sections whose resistances are $250 \mathrm{ohms}$ or more are measured in the network of figure $4 \mathrm{~b}$. (The rod resistances are expected to be negligible compared with the resistance of the section to which they are assigned.) The guard circuit of the particular standard volt box under discussion is not equipped with taps below the $15-\mathrm{v}$ range so that it is used only on the ranges of $15 \mathrm{v}$ and higher. Thus, for all successive resistances measured after the first five and to, but not including, the " 15 to 30 " section, the guard branch of figure $4 \mathrm{~b}$ is not used. For all remaining measurements the guard branch is connected as shown in the figure.

The appropriate bridge network is balanced by adjusting the dials of the DRRS for a null on the detector as each section, or summation of sections, beginning with $R_{1,1}$ is inserted in succession in the "unknown" arm of the bridge. The procedure is as follows. With $S=S_{a 1}=50$ ohms, the bridge of figure $4 \mathrm{a}$ is balanced with the first 50-ohm section

\footnotetext{
4 The resistance per section of the guard of the volt box is 0.8 that of the working resistance.

The influence of these rod resistances can be completely avoided by modifying the circuit so that the $50-0 h m$ sections are measured as 4 -terminal resistors.
} This technique is described in reference [2].

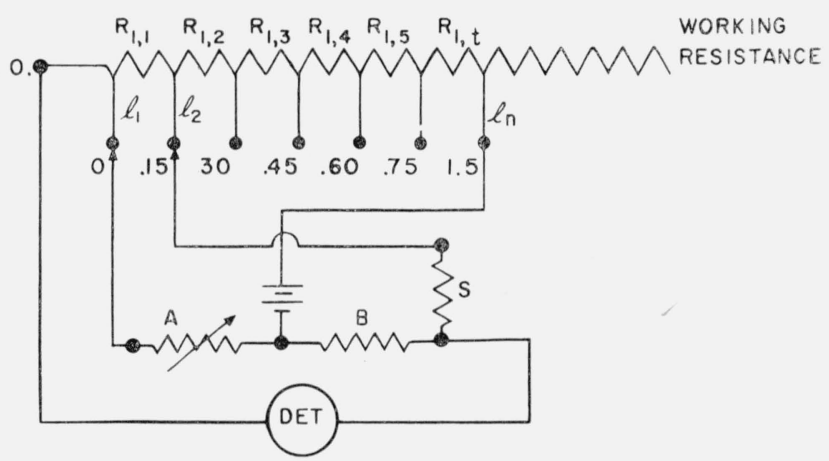

Figure 4a. Circuit for measuring the lower-valued ratios.

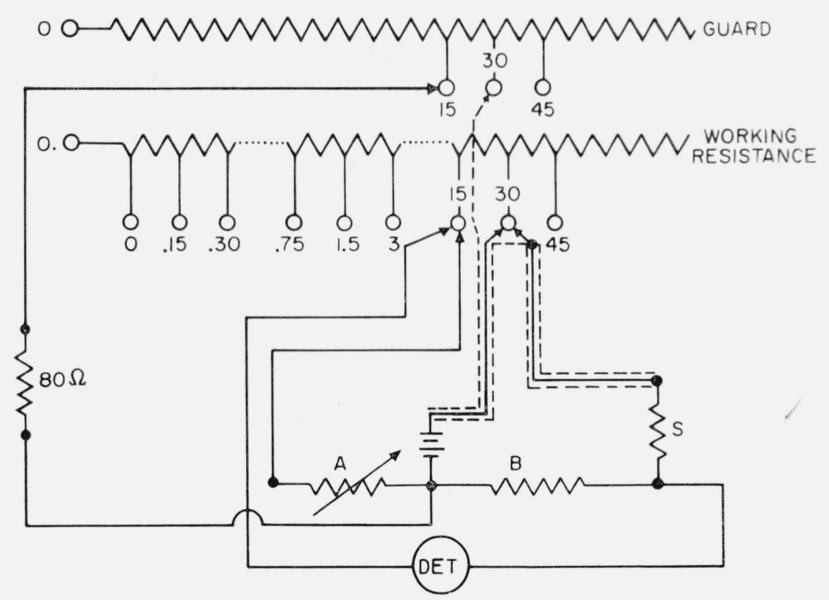

Figure 4b. Circuit for measuring the higher-valued ratios. 
$\left(R_{1,1}\right)$ inserted in the unknown arm. In rapid sequence, each of the remaining four 50 -ohm sections is inserted in turn and the bridge rebalanced in each instance. The resistance of each $50-\mathrm{ohm}$ section can now be stated in terms of the resistance of $R_{1,1}$ and the measured difference, and the group can be summed to give $\sum_{k=1}^{k=5} R_{1, k}$ (computed). The next set of balances completes the series of measurements required for group $M_{1}$. It consists of two measurements using the appropriate circuit of figure $4 \mathrm{~b}$ and with $S=S_{b 1}=250$ ohms. The reference balance is made with the sum of the 50 -ohm sections $\sum_{k=1}^{k=5} R_{1, k}$, having a nominal value of 250 ohms, connected into the unknown arm through the zero and 0.75 binding posts and is immediately followed by a measurement of $R_{1, t}$. This set of measurements provides a value of $R_{1, i}$ (sixth section) in terms of $\sum_{k=1}^{k=5} R_{1, k}$ and the measured difference. Since the value of $\sum_{k=1}^{k=5} R_{1, k}$ is obtained by summing the values from the first five measurements, straightforward substitution gives the value of $R_{1, t}$ in terms of $R_{1,1}$ and the sum of the measured differences. Adding this value of $R_{1, t}$ to the value of $\sum_{k=1}^{k=5} R_{1, k}$ (computed) gives the total resistance of group $M_{1}$ referred to the reference section $R_{1,1}$. These two sets of measurements expressed mathematically are as follows:

$$
\begin{aligned}
& R_{1,1}=S_{a 1}\left[1+\left(D_{1,1}-D_{0}\right)\right] \\
& R_{1,2}=S_{a 1}\left[1+\left(D_{1,2}-D_{0}\right)\right] \\
& R_{1,3}=S_{a 1}\left[1+\left(D_{1,3}-D_{0}\right)\right] \\
& R_{1,4}=S_{a 1}\left[1+\left(D_{1,4}-D_{0}\right)\right] \\
& R_{1,5}=S_{a 1}\left[1+\left(D_{1,5}-D_{0}\right)\right]
\end{aligned}
$$

where $\left(D_{1,1}-D_{0}\right),\left(D_{1,2}-D_{0}\right)$, etc., are the differences in proportional parts ${ }^{6}$ as deduced from the readings of the DRRS; $D_{0}$ is the reading of the DRRS for an exact $1: 1$ ratio. (The significance of $D_{0}$ is explained in appendix A.)

Subtracting the first equation from each succeeding equation and from itself, gives

$$
\begin{aligned}
R_{1,1} & =R_{1,1} \\
R_{1,2} & =R_{1,1}+\mathrm{S}_{a 1} d_{1,2} \\
R_{1,3} & =R_{1,1}+S_{a 1} d_{1,3} \\
R_{1,4} & =R_{1,1}+S_{a 1} d_{1,4} \\
R_{1,5} & =R_{1,1}+S_{a 1} d_{1,5} \\
\sum_{k=1}^{k=5} R_{1, k} & =5 R_{1,1}+S_{a 1} \sum_{k=1}^{k=5} d_{1, k}
\end{aligned}
$$

6 See appendix A. where $d_{1,2}=\left(D_{1,2}-D_{1,1}\right) ; d_{1,3}=\left(D_{1,3}-D_{1,1}\right)$ etc.

Since $S_{a 1}=R_{1,1}$ closel y enough,

$$
\sum_{k=1}^{k=5} R_{1, k}=5 R_{1,1}\left[1+\sum_{k=1}^{k=5} \frac{d_{1, k}}{5}\right]
$$

The second set of measurements stated in similar fashion is

$$
\begin{aligned}
\sum_{k=1}^{k=5} R_{1, k} & =S_{b 1}\left[1+\left(D_{1, s}-D_{0}\right)\right] \\
R_{1, t} & =S_{b 1}\left[1+\left(D_{1, t}-D_{0}\right)\right] .
\end{aligned}
$$

On subtracting, this gives

$$
R_{1, t}=\sum_{k=1}^{k=5} R_{1, k}+S_{b 1} d_{1, t} \text { where } d_{1, t}=\left(D_{1, t}-D_{1, s}\right) .
$$

Substituting eq (6) in the above expression and remembering that

$S_{b 1}=5 R_{1,1}$ closely enough, $R_{1, t}$ becomes

$$
R_{1, t}=5 R_{1,1}\left[1+\sum_{k=1}^{k=5} \frac{d_{1, k}}{5}+d_{1, t}\right] \text {. }
$$

Adding eqs (6) and (7) and arranging terms gives

$$
\sum_{k=1}^{k=5} R_{1, k}+R_{1, t}=10 R_{1,1}\left[1+\sum_{k=1}^{k=5} \frac{d_{1, k}}{5}+\frac{d_{1, t}}{2}\right]
$$

which states the total resistance of group $M_{1}$ in terms of $R_{1,1}$ and the measured differences.

Group $M_{1}$, having a nominal value of $500 \mathrm{ohms}$ and a true value given by eq (8), serves as the first section of group $M_{2}$ which, as in the case of group $M_{1}$, consists of five nominally equal sections plus a sixth section having a value nominally equal to the sum of the five sections (see fig. 3). The sequence of seven balances is now obtained for group $M_{2}$. In the first set of this sequence, with $S=S_{a 2}=500$ ohms, each of the $500 \mathrm{ohm}$ sections, beginning with group $M_{1}$ as the reference section, is inserted in the bridge to obtain five measurements. In the second set two balances are obtained, first with all 10 sections $(2,500 \mathrm{ohms})$ in the unknown arm and then with the eleventh section (2,500 ohms) inserted; for this set, $S=S_{b 2}=2,500$ ohms. Since group $M_{1}$ has a computed value given by eq (8), the summation procedure can be extended through group $M_{2}$ which contains all sections up to the $15 \mathrm{v}$ tap and becomes the first section of $M_{3}$.

The same procedure is carried forward through groups $M_{3}$ and $M_{4}$ with resistance $S$ always having a value nominally equal to that of the section being measured.

The mathematical treatment presented in this section attempts to clarify the significance of the computation process associated with the step-up procedure (sometimes called a bootstrap technique), which may be masked in the more formal approach given in appendix $\mathrm{B}$. 


\subsection{Computations}

The readings of the DRRS having been recorded, the summation of their differences can be computed according to the following equation (developed in appendix B) to give the correction to the nominal ratios of the standard volt box

$$
\begin{array}{r}
N_{m, k}=N_{m, k}^{\prime}\left[1+\frac{\sum_{k=1}^{k=5} d_{1, k}+\sum_{k=1}^{k=5} d_{2, k} \ldots+\sum_{k=1}^{k=5} d_{m-1, k}}{5}\right. \\
\left.+\frac{d_{1, t}+d_{2, t} \ldots+d_{m-1, t}}{2}+\frac{\sum_{k^{\prime}=1}^{k^{\prime}=k} d_{m, k^{\prime}}}{k}\right] .
\end{array}
$$

Where $N_{m, k}=$ true value of the ratios,

$N_{m, k}^{\prime, k}=$ nominal values of the ratios;

$d$ 's $=$ measured differences in proportional parts.

The schedule of computation given in table 1 was derived, in effect, by applying eq (9) to successively increasing values of $k$ and $m$. Its arrangement permits the computation of all corrections in a single process without having to apply the equation directly each time a ratio is determined. The summation of the differences within a set (five-set or two-set) are kept separate until the final manipulation. This is indicated by columns 4,5 , and 6 . The $d$ 's are the differences within each set; the q's are the corresponding summations; and the $r$ 's refer to the separate quantities appearing in the final terms of eq (9). Column 7 is the summation of the sets for each successive ratio.

\section{Test Console}

After the test method was investigated for more than a year with the circuit assembled in "breadboard" fashion, a small test console was designed and built so that the set-up time for a test would be reduced to a minimum. The console, shown in the photograph and indicated in figure 5 , houses the DRRS, the galvanometer with its damping resistance, a mercury stand to accommodate the standard resistors, the 80 -ohm guard resistor and the battery reversing switch. The DRRS, located in the lower portion of the console, is connected to the circuit by short leads brought to three binding posts located on the vertical panel immediately above the terminals of the DRRS. (With this arrangement the DRRS can be easily removed and made available for other work.) The detector is a portable galvanometer mounted on a platform within the enclosure immediately above and to the left of the DRRS. The galvanometer scale is observed through an opening in the front panel and the damping resistance, permanently mounted on the inside, is adjustable in steps by a knob accessible to the operator. The mercury stand, located within the console on a platform adjacent to the detector and above the DRRS, can accommodate either a single resistor or several arranged in either series or parallel combination. A removable top permits ready access to all components and wiring.

As shown in the wiring diagram of figure 5, the battery reversing switch, accessible on the front panel, carries a two-conductor shielded cable that terminates at a fitting located on the side panel.

\begin{tabular}{|c|c|c|c|c|c|c|c|}
\hline 1 & 2 & 3 & 4 & 5 & 6 & 7 & 8 \\
\hline $\begin{array}{l}\text { Section in } \\
\text { bridge } \\
\text { arm }\end{array}$ & $\underset{S}{\text { Resistor }}$ & $\begin{array}{l}\text { Reading of } \\
\text { DRRS }\end{array}$ & $d^{\prime} s$ & $\begin{aligned} & q^{\prime} s \\
= & \Sigma d^{\prime} s\end{aligned}$ & $\begin{aligned} & r^{\prime} s \\
& \Sigma d^{\prime} s \\
& k\end{aligned}$ & $\begin{array}{l}\text { Correction referred to } \\
0-0.15 \text { section }\end{array}$ & Range \\
\hline $\begin{array}{r}0-0.15 \\
0.15-0.30 \\
.30-0.45 \\
.45-0.60 \\
.60-0.75\end{array}$ & $\begin{array}{r}\text { Ohms } \\
50 \\
50 \\
50 \\
50 \\
50\end{array}$ & $\begin{array}{l}D_{1,1} \\
D_{1,2} \\
D_{1,3} \\
D_{1,4} \\
D_{1,5}\end{array}$ & $\begin{array}{l}d_{1,1}=D_{1,1}-D_{1,1}=0 \\
d_{1,2}=D_{1,2}-D_{1,1} \\
d_{1,3}=D_{1,3}-D_{1,1} \\
d_{1,4}=D_{1,4}-D_{1,1} \\
d_{1,5}=D_{1,5}-D_{1,1}\end{array}$ & $\begin{array}{l}q_{1,2}=d_{1,2} \\
q_{1,3}=q_{1,2}+d_{1,3} \\
q_{1,4}=q_{1,3}+d_{1,4} \\
q_{1,5}=q_{1,4}+d_{1,5}\end{array}$ & $\begin{array}{l}r_{1,2}=1 / 2 q_{1,2} \\
r_{1,3}=1 / 3 q_{1,3} \\
r_{1,4}=1 / 4 q_{1,4} \\
r_{1,5}=1 / 5 q_{1,5}\end{array}$ & $\begin{array}{ll} & \text { ppm } \\
\mu_{1,1}=0 & \\
\mu_{1,2}=r_{1,2} \\
\mu_{1,3}=r_{1,3} \\
\mu_{1,4}=r_{1,4} \\
\mu_{1,5}=r_{1,5}\end{array}$ & $\begin{array}{l}0-0.15 \\
0-0.30 \\
0-0.45 \\
0-0.60 \\
0-0.75\end{array}$ \\
\hline $\begin{array}{r}0-0.75 \\
0.75-1.5\end{array}$ & $\begin{array}{l}250 \\
250\end{array}$ & $D_{1,8}$ & $\begin{array}{l}d_{1,8}=D_{1,8}-D_{1,8}=0 \\
d_{1, t}=D_{1, t}-D_{1, s}\end{array}$ & $q_{1, t}=d_{1, t}$ & $r_{1}=1 / 2 q_{1}$ & $\mu_{2}=\mu_{15}+r_{1}$ & $0-1-5$ \\
\hline $0-1.5$ & 500 & $D_{2,1}$ & $d_{2,1}=D_{2,1}-D_{2,1}=0$ & & & & \\
\hline $\begin{array}{l}\text { 1. } 5-3.0 \\
3.0-4.5 \\
\text { 4. } 5-6.0 \\
6.0-7.5\end{array}$ & $\begin{array}{l}500 \\
500 \\
500 \\
500\end{array}$ & $\begin{array}{l}D_{2,2} \\
D_{2,3} \\
D_{2,4} \\
D_{2,5}\end{array}$ & $\begin{array}{l}d_{2,2}=D_{2,2}-D_{2,1} \\
d_{2,3}=D_{2,3}-D_{2,1} \\
d_{2,4}=D_{2,4}-D_{2,1} \\
d_{2,5}=D_{2,5}-D_{2,1}\end{array}$ & $\begin{array}{l}q_{2,2}=d_{2,2} \\
q_{2,3}=q_{2,2}+d_{2,3} \\
q_{2,4}=q_{2,3}+d_{2,4} \\
q_{2,5}=q_{2,4}+d_{2,5}\end{array}$ & $\begin{array}{l}r_{2,2}=1 / 2 q_{2,2} \\
r_{2,3}=1 / 3 q_{2,3} \\
r_{2,4}=1 / 4 q_{2,4} \\
r_{2,5}=1 / 5 q_{2,5}\end{array}$ & $\begin{array}{l}\mu_{2,2}=\mu_{2,1}+r_{2,2} \\
\mu_{2,3}=\mu_{2,1}+r_{2,3} \\
\mu_{2,4}=\mu_{2,1}+r_{2,4} \\
\mu_{2,5}=\mu_{2,1}+r_{2,5}\end{array}$ & $\begin{array}{l}0-3.0 \\
0-4.5 \\
0-6.0 \\
0-7.5\end{array}$ \\
\hline $0-7.5$ & 2,500 & $D_{2,8}$ & $d_{2,8}=D_{2,8}-D_{2,8}=0$ & & & & \\
\hline 7. $5-15$ & 2,500 & $D_{2, t}$ & $d_{2, t}=D_{2, t}-D_{2, s}$ & $q_{2, t}=d_{2, t}$ & $r_{2, t}=1 / 2 q_{2, t}$ & $\mu_{3,1}=\mu_{2,5}+r_{2, t}$ & $0-15$ \\
\hline $\begin{array}{r}0-15 \\
15-30 \\
30-45 \\
45-50 \\
60-75\end{array}$ & $\begin{array}{l}5,000 \\
5,000 \\
5,000 \\
5,000 \\
5,000\end{array}$ & $\begin{array}{l}D_{3,1} \\
D_{3,2} \\
D_{3,3} \\
D_{3,4} \\
D_{3,5}\end{array}$ & $\begin{array}{l}d_{3,1}=D_{3,1}-D_{3,1}=0 \\
d_{3,2}=D_{3,2}-D_{3,1} \\
d_{3,3}=D_{3,3}-D_{3,1} \\
d_{3,4}=D_{3,4}-D_{3,1} \\
d_{3,5}=D_{3,5}-D_{3,1}\end{array}$ & $\begin{array}{l}q_{3,2}=d_{3,2} \\
q_{3,3}=q_{3,2}+d_{3,3} \\
q_{3,4}=q_{3,3}+d_{3,4} \\
q_{3,5}=q_{3,4}+d_{3,5}\end{array}$ & $\begin{array}{l}r_{3,2}=1 / 2 q_{3,2} \\
r_{3,3}=1 / 3 q_{3,3} \\
r_{3,4}=1 / 4 q_{3,4} \\
r_{3,5}=1 / 5 q_{3,5}\end{array}$ & $\begin{array}{l}\mu_{3,2}=\mu_{3,1}+r_{3,2} \\
\mu_{3,3}=\mu_{3,1}+r_{3,3} \\
\mu_{3,4}=\mu_{3,1}+r_{3,4} \\
\mu_{3,5}=\mu_{3,1}+r_{3,5}\end{array}$ & $\begin{array}{l}0-30 \\
0-45 \\
0-60 \\
0-75\end{array}$ \\
\hline $0-75$ & 25,000 & $D_{3,8}$ & $d_{3,8}=D_{3,8}-D_{3,8}=0$ & & & & \\
\hline $\begin{array}{r}75-150 \\
0-150\end{array}$ & $\begin{array}{l}25,000 \\
50,000\end{array}$ & $\begin{array}{l}D_{3, t} \\
D_{4,1}\end{array}$ & $\begin{array}{l}d_{3, t}=D_{3, t}-D_{3,8} \\
d_{4,1}=D_{4,1}-D_{4,1}=0\end{array}$ & $q_{3, t}=d_{3, t}$ & $r_{3, t}=1 / 2 q_{3, t}$ & $\mu_{4,1}=\mu_{3,5}+r_{3, t}$ & $0-150$ \\
\hline $\begin{array}{l}150-300 \\
300-450 \\
450-600 \\
600-750\end{array}$ & $\begin{array}{l}50,000 \\
50,000 \\
50,000 \\
50,000\end{array}$ & $\begin{array}{l}D_{4,2} \\
D_{4,3} \\
D_{4,4} \\
D_{4,5}\end{array}$ & $\begin{array}{l}d_{4,2}=D_{4,2}-D_{4,1} \\
d_{4,3}=D_{4,3}-D_{4,1} \\
d_{4,4}=D_{4,4}-D_{4,1} \\
d_{4,5}=D_{4,5}-D_{4,1}\end{array}$ & $\begin{array}{l}q_{4,2}=d_{4,2} \\
q_{4,3}=q_{4,2}+d_{4,3} \\
q_{4,4}=q_{4,3}+d_{4,4} \\
q_{4,5}=q_{4,4}+d_{4,5}\end{array}$ & $\begin{array}{l}r_{4,2}=1 / 2 q_{4,2} \\
r_{4,3}=1 / 3 q_{4,3} \\
r_{4,4}=1 / 4 q_{4,4} \\
r_{4,5}=1 / 5 q_{4,5}\end{array}$ & $\begin{array}{l}\mu_{4,2}=\mu_{4,1}+r_{4,2} \\
\mu_{4,3}=\mu_{4,1}+r_{4,3} \\
\mu_{4,4}=\mu_{4,1}+r_{4,4} \\
\mu_{4,5}=\mu_{4,1}+r_{4,5}\end{array}$ & $\begin{array}{l}0-300 \\
0-450 \\
0-600 \\
0-750\end{array}$ \\
\hline
\end{tabular}

TABLE 1. Schedule for computing corrections for the standard volt box 

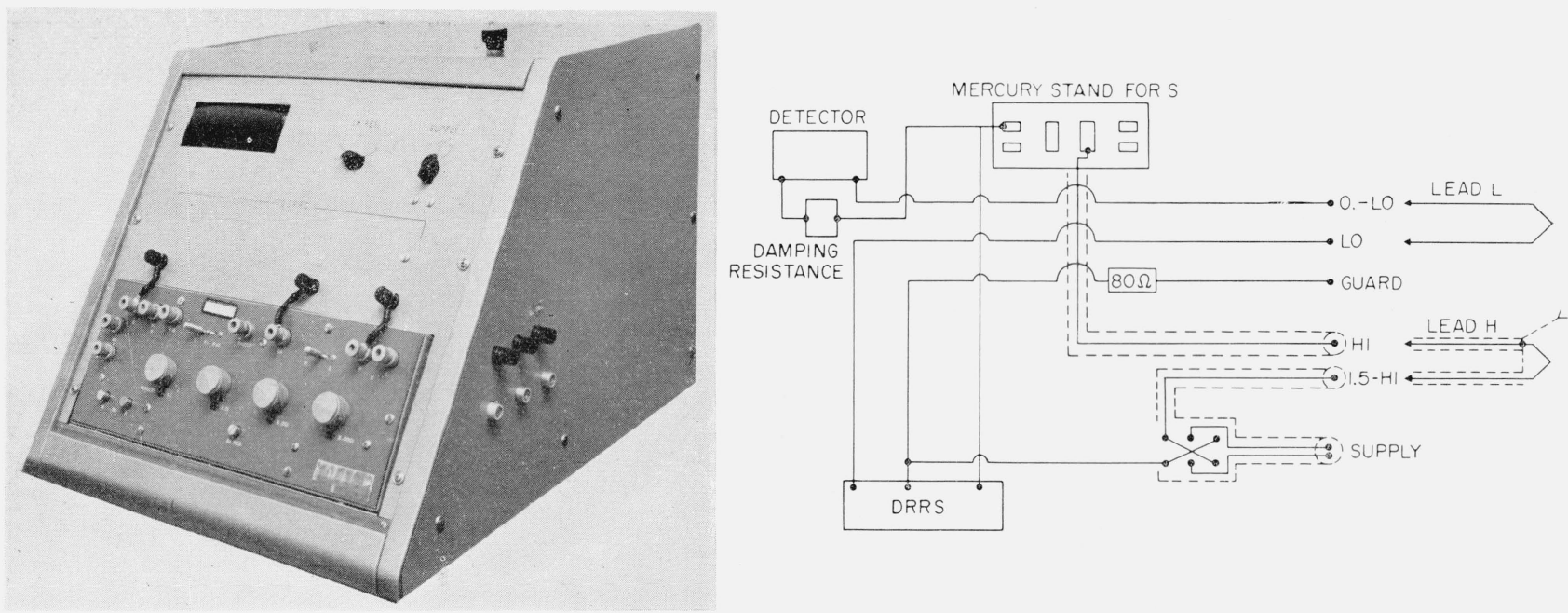

Figure 5. Test console and layout.

An external d-c supply is connected to this fitting with a pair of shielded leads equipped with a mating connector. The side panel also carries two singleconductor coax fittings, identified as "1.5-Hi" and "Hi," and three binding posts, identified as "0.-Lo," "Lo,", and "Guard."

When the first five sections of the standard volt box are measured separate unshielded leads are used to connect the terminals of the volt box $(1.5$ post, high-potential post of the section being measured, 0 . post, and low-potential post of the section being measured) to the respective terminals of the console (1.5-Hi, Hi, 0.-Lo, and Lo). For all other measurements special leads $H$ and $L$ are used to connect the section under test as indicated in figure 5. When the guard of the volt box is used, the pigtail of lead $H$ is connected to the high-potential terminal of the guard section and a connection is made from the "Guard" binding post to the low-potential terminal of the guard section. Otherwise, these last two connections are omitted.

\section{Consideration of Errors}

An examination of errors requires a careful study of all known effects that could render a measured value different from the true value within the specified accuracy. An appraisal of all components of the measuring circuit as well as a justification of approximations must be included, even though in this instance one would expect the associated errors to be negligible.

The first possible source of error to consider is the DRRS. As in the usual case, this type of error can be avoided by calibrating the instrument and applying corrections. Apart from this procedure, however, it should be emphasized that an error in the measured value is diluted when the values of the ratios are considered. For example, if each scale reading were in error by $\epsilon=1 \mathrm{ppm}$ and all errors were in such a direction as to add when computing each difference their contribution to the error of a given ratio, when summed within any one of the five-sets, would be

$$
\frac{(k-1) 2 \epsilon}{k}=\frac{2(k-1)}{k}
$$

where $k$ is the number of summed sections. For $k=5$ the error would be $8 / 5=1.6 \mathrm{ppm}$. Similarly, the error introduced in the measurements of the two-sets would be

$$
\frac{2 \epsilon}{2}=1 \mathrm{ppm}
$$

The maximum error would occur for the highest range since it contains all the sets. This would amount to only $9.4 \mathrm{ppm}$ under these adverse conditions. Since the corrections to the DRRS used at NBS are less than $0.3 \mathrm{ppm}$ and their signs and magnitudes have a random distribution, the errors in the ratio measurements are almost certain to be less than $1 \mathrm{ppm}$ with no corrections applied to the dial readings.

A source of error is concealed in the base (or balance) equation used in appendix B to develop the general expression for the volt box ratios. Referring to appendix $\mathrm{A}$, the exact base equation is given as

$$
R_{m, k}=S\left[1+\frac{1}{1+\frac{l_{2}}{B_{m}}}\left(D_{m, k}-D_{0}\right)\right]
$$

whereas, the approximate value used in the mathematical development in appendix B is

$$
R_{m, k}=S\left[1+\left(D_{m, k}-D_{0}\right)\right] .
$$

The latter departs from exactness by an amount $\frac{l_{2}}{B_{m}}\left(D_{m, k}-D_{0}\right)$ but is of second order. For example, if $\frac{l_{2}}{B_{m}}$ were as large as 0.1 percent and $\left(D_{m, k}-D_{0}\right)$ rep- 
resented a change of $500 \mathrm{ppm}$ in the $A$-arm, the value for $R_{m, k}$ would be in error by only $0.5 \mathrm{ppm}$.

Another error is concealed through approximation in developing the general expression that defines the volt box ratios (appendix B) when it is assumed that the value of the "Dummy" resistor, including leads, is exactly equal to that of the section being measured. The error introduced through this assumption is second order since it is the product of two small terms. For example, consider the summation of the first five sections (refer to eq (6)).

$$
\sum_{k=1}^{k=5} R_{1, k}=5 R_{1,1}+S_{a 1} \sum_{k=1}^{k=5} d_{1, k}
$$

If $S_{a 1}$ differs from $R_{1.1}$ and is given by $S_{a 1}=R_{1,1}$ $\left[1+\mu_{1}\right]$ the summation becomes

$$
\sum_{k=1}^{k=5} R_{1, k}=5 R_{1,1}\left[1+\sum_{k=1}^{k=5} \frac{d_{1, k}}{5}+\mu_{1} \sum_{k=1}^{k=5} \frac{d_{1, k}}{5}\right]
$$

where the magnitude of the error is $\mu_{1} \sum_{k=1}^{k=5} \frac{d_{1 . k}}{5}$.

If both $\mu_{1}$ and the summation were as large as 500 ppm, the error would be only $0.05 \mathrm{ppm}$.

Another possible source of error arises if the resistance in any one arm changes during the measurements of a given set. This change could occur in the leads and contacts, for example, and its effect would be greatest when measuring the 50 -ohm sections. A change of this nature can be considered as a change in $D_{0}$ and an estimate of the error it introduces in a measured ratio can be obtained by considering the measurements of the first set. Assume an extreme case in which the resistance of a given arm increases in equal increments after the first measurement so that for subsequent balances $D_{0}$ takes on new values. The measured values for the set become

$$
\begin{aligned}
& R_{1,1}=S_{a 1}\left[1+\left(D_{1,1}-D_{0}\right)\right] \\
& R_{1,2}=S_{a 1}\left[1+D_{1,2}-\left(D_{0}+\epsilon\right)\right] \\
& R_{1,3}=S_{a 1}\left[1+D_{1,3}-\left(D_{0}+2 \epsilon\right)\right] \\
& R_{1,4}=S_{a 1}\left[1+D_{1,4}-\left(D_{0}+3 \epsilon\right)\right] \\
& R_{1,5}=S_{a 1}\left[1+D_{1,5}-\left(D_{0}+4 \epsilon\right)\right]
\end{aligned}
$$

where $\epsilon$ is the change in $D_{0}$ between successive balances. After taking differences, any given sum within the set can be written as

$$
\sum_{k^{\prime}=1}^{k^{\prime}=k} R_{1, k^{\prime}}=k R_{1,1}\left[1+\sum_{k^{\prime}=1}^{k^{\prime}=k} \frac{d_{1}, k^{\prime}}{k}-\sum_{k^{\prime}=1}^{k^{\prime}=k} \frac{\left(k^{\prime}-1\right) \epsilon}{k}\right] .
$$

Thus an error in any ratio formed within the set is $\sum_{k^{\prime}=1}^{k^{\prime}=k} \frac{\left(k^{\prime}-1\right) \epsilon}{k}$. For example, if a lead resistance increased in increments of $0.0001 \mathrm{ohm}$ in the $S$-arm (50 ohms) the magnitude of the error introduced in the ratio $\frac{\sum_{k=1}^{k=5} R_{1, k}}{R_{1,1}}$ would be $4 \mathrm{ppm}$. If the usual precautions are taken, the probability of this kind of an occurrence is quite remote and could be easily observed from the detector response. A single, abrupt change would be more difficult to detect but an error from this cause can be avoided by taking a repeat set of measurements.

The final consideration involves those errors that arise from the unavoidable presence of the rod resistances. This source of error could be the most critical of any because of its insidious nature. Two errors must be examined when considering the rod resistances. If the rod resistances are equal they can introduce an error if, when the standard volt box is used, the connections differ from those made during the process of calibration. The second error occurs if the rod resistances differ in magnitude.

Consider the cause of the first error. When in use, the connections to the standard volt box are as shown in figure 6 . There are no currents in rods $l_{1}$ and $l_{2}$; rod $l_{n}$, however, carries current by virtue of its connection to the supply. Thus the ratio of the standard in use is

$$
N_{s}=\frac{\sum_{1}^{n} R_{n^{\prime}}+l_{n}}{R_{1,1}} .
$$

When the first five sections are measured (refer to fig. $4 \mathrm{a}$ ) the rods are in the $A$ - and $S$-arms. Thus, if the ratio under consideration were one confined solely to these sections, the measured ratio would be

$$
N_{s}^{\prime}=\frac{\sum_{1}^{n} R_{n^{\prime}}}{R_{1,1}}
$$

and eq (10) can be written as

$$
N_{s}=N_{s}^{\prime}+\frac{l_{n}}{R_{1,1}}=N_{s}^{\prime}\left[1+\frac{l_{n}}{N_{s}^{\prime} R_{1,1}}\right]
$$

In the measurement and summing process through the higher ranges, the rod resistances form part of the section being measured. (See fig. 4b.) However, if the rod resistances are equal, their effects

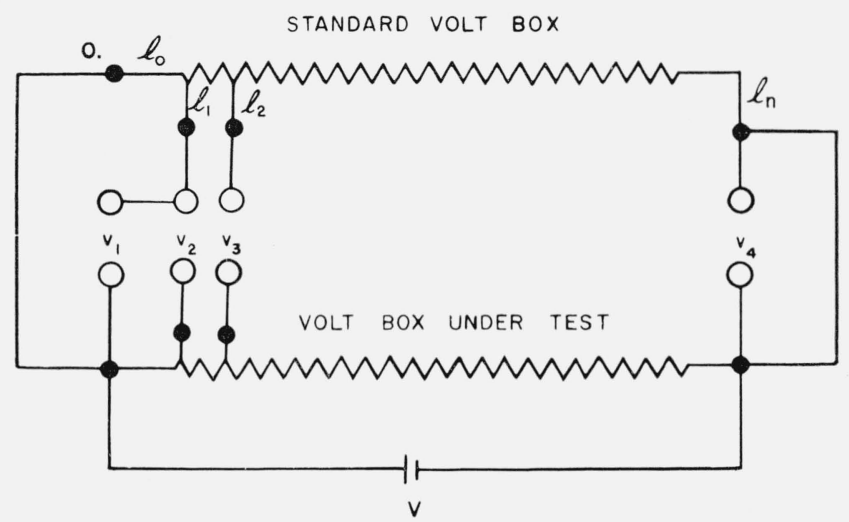

Figure 6. Circuit emphasizing one effect from rod resistances. 
cancel and the difference between the ratio in use and the one measured is the same as that given in eq (12). It is apparent then that the ratio in use differs from the measured ratio by an amount equal to the rod resistance divided by the total resistance of the range used and occurs even though the rod resistances are equal. If the standard volt box were used as a 4-terminal voltage divider the above correction to the ratio in use would be $\frac{l_{n}+l_{0}}{N_{s}^{\prime} R_{1,1}}$ since $l_{0}$ becomes part of the high voltage side.

The magnitude of an error arising from inequalities in the rod resistances depends on where the rods are located in the bridge network. As stated in section 4.2 , there are three alternatives for connecting the rods and the choice of orientation is not as clearly indicated when measuring the $50-\mathrm{ohm}$ sections as when measuring the higher-valued sections. For the purpose of analysis, consider the measurement of the low-valued sections and the particular connection shown in figure $4 \mathrm{a}$.

For this condition, differences among the rod resistances may be considered as producing changes in $D_{0}$ so that, if the respective rod resistances are identified as $l_{1}, l_{2}$, etc., the measured resistances are

$$
\begin{aligned}
& R_{1,1}=S_{a 1}\left[1+D_{1,1}-D_{0}\right] \\
& R_{1,2}=S_{a 1}\left[1+D_{1,2}-D_{0}+\frac{l_{2}-l_{1}}{A}+\frac{l_{3}-l_{2}}{S_{a 1}}\right] \\
& R_{1,3}=S_{a 1}\left[1+D_{1,3}-D_{0}+\frac{l_{3}-l_{1}}{A}+\frac{l_{4}-l_{2}}{S_{a 1}}\right] \\
& R_{1,4}=S_{a 1}\left[1+D_{1,4}-D_{0}+\frac{l_{4}-l_{1}}{A}+\frac{l_{5}-l_{2}}{S_{a 1}}\right] \\
& R_{1,5}=S_{a 1}\left[1+D_{1,5}-D_{0}+\frac{l_{5}-l_{1}}{A}+\frac{l_{6}-l_{2}}{S_{a 1}}\right] .
\end{aligned}
$$

If the rod resistances are measured, their contribution to the error of any ratio can be evaluated. Comparable sets of equations can be formed for the other two cases and corresponding errors evaluated. If the successive values of rod resistances neither increase nor decrease, it cannot be stated categorically that one connection is preferred to another. Thus a comparison of the resulting errors for the three possible connections must be made and the connection chosen which yields the smallest errors. Such a comparison applied to the NBS standard volt box indicated that the circuit of figure $4 \mathrm{a}$ is preferred. As measurements are continued through the higher ranges, the rods are connected as part of the large resistance being measured so that their effects are further reduced.

In the NBS standard volt box recently acquired, rod resistances from zero through the $6-\mathrm{v}$ range are about $5 \times 10^{-4} \mathrm{ohm}$ and their differences are sufficiently small and random in sign that the maximum error they produce in a ratio measurement is less than $0.3 \mathrm{ppm}$.

\section{Summary}

A particular type of voltage ratio standard and a method for its calibration are discussed. Although the method is described in its particular application to the NBS standard it can be used equally well in other networks. Errors that might appear in the measurement of its ratios and procedures for evaluating their magnitudes are outlined. With a standard volt box of proper design, the calibration method as set forth should yield results that are good to an accuracy of $10 \mathrm{ppm}$ or better. This estimate of accuracy is based on the theoretical considerations of the circuit and its parameters, on the continued good agreement with those values of ratio obtained by the older method, and on other self-consistent tests.

The author appreciates the assistance of Rita McAuliff and Ronald Dziuba who made most of the measurements and constructed the test console.

\section{Appendix $\bar{A}$}

\subsection{Comments on the DRRS}

Complete discussions on the DRRS and its use in the accurate measurement of $\mathrm{d}-\mathrm{c}$ resistance are contained in references [2], [3], and [4] so that an elaborate treatment is not warranted. However, certain aspects are emphasized here in order to clarify the meaning of particular equations, as well as justify their use.

The DRRS is used principally in comparing two nearly equal resistances and under these conditions has an accuracy of about $1 \mathrm{ppm}$. It comprises two resistance arms of about equal magnitudes with one arm $B^{\prime}$ having a single-valued resistance (in most cases 100 or $1,000 \mathrm{ohms}$ ) and the other consisting of a fixed resistance in series with an adjustable section. The adjustable branch contains, in most cases, four resistance groups, each provided with a selector to vary the resistance and register the change on suitably marked dials. The resistance of the $A^{\prime}$-arm can be varied by about 0.5 percent on either side of the $B^{\prime}$-arm value to a least count of $1 \mathrm{ppm}$. The dials register in increasing numbers as resistance is added to the fixed portion of the $A^{\prime}$-arm. Since the DRRS is used to measure differences between two nearly equal quantities, the dial readings can be translated to ppm or, as is often the case, the dials may be marked in ppm.

The reader should note carefully the form in which small corrections are recorded or used in derivations and computations. As usually constructed, a change of one step on the lowest dial of a four-dial DRRS corresponds to a change of $1 \mathrm{ppm}$ in the ratio. At one particular balance of a measuring circuit the reading of the DRRS should be thought of simply as a four-digit number, such as 5472. If a second balance of the same circuit yields a DRRS reading of 5459 the measured ratio change is $-13 \mathrm{ppm}$, this 
being the first point at which the abbreviation "ppm" should be introduced. In recording data and computations (see table 1) it is convenient and space saving to enter DRRS readings as four-digit numbers, and ratio changes in ppm. In deriving equations, however, (and corrections based thereon) ratio changes should be expressed in proportional parts. A ratio change in proportional parts is one millionth of the change expressed in $\mathrm{ppm}$. In the above illustration the ratio change is -0.000013 proportional parts. See reference [2] for further discussion of these terms.

\subsection{Bridge Balance Equation}

Figure 7 indicates a bridge network in which the adjustable arm of the DRRS is represented as a section of fixed resistance in series with a uniform slide wire of high resolution. Let $B_{m}$ indicate the resistance of the $B$-arm of the DRRS, $A_{m}$ the resistance of its $A$-arm for which $\overline{A_{m}=B_{m}}, l_{2}$ and $l_{1}$ the respective lead resistances with $l_{2} \neq l_{1}$, and $S$ the resistance of a standard serving as the "Dummy" resistor. For convenience, let the DRRS be without error as previously determined from calibration.

Assume first that a standard resistor $S_{1}$, having a resistance equal to $S$, is inserted in the unknown arm and the bridge balances at some point for which the $A$ - and $B$-arms of the DRRS are not equal. Let the resistance of the $A$-arm of the DRRS be $A_{0}$ for this balance condition. The equation of balance is given by

$$
S=S\left[\frac{\left(A_{0}+l_{1}\right)}{\left(B_{m}+l_{2}\right)}\right]
$$

Thus $A_{0}$ is the resistance of the $A$-arm of the DRRS when the ratio arms ( $A$ - and $B$-) of the bridge are in

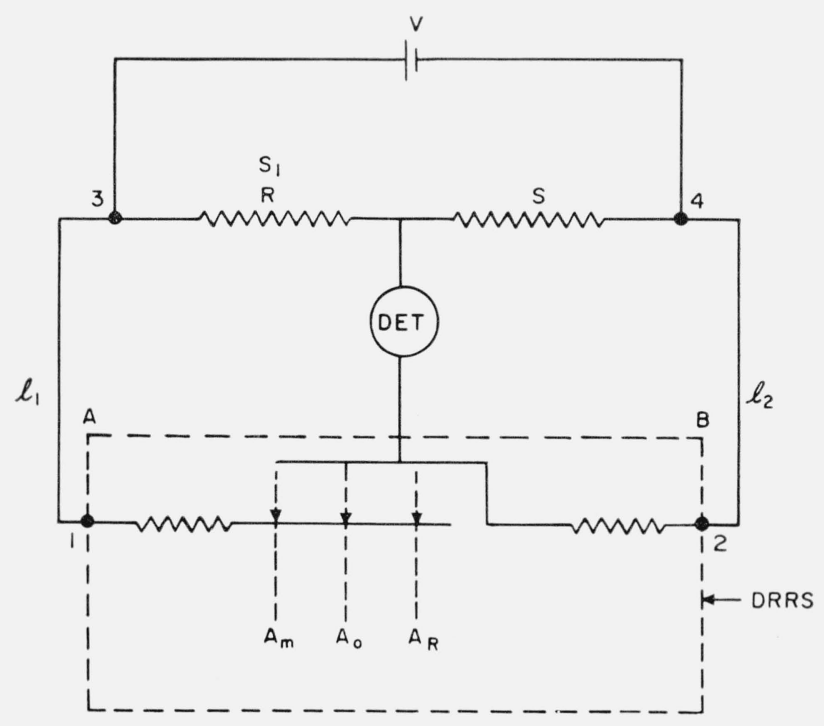

FIGURE 7. Circuit indicating the DRRS with respect to the balance equation. a 1:1 ratio. Next, let $S_{1}$ be replaced by a resistance whose value is $R$ and let $A_{R}$ be the resistance of the $A$-arm of the DRRS under the new balance condition. The equation of balance is

$$
R=S\left[\frac{\left(A_{R}+l_{1}\right)}{\left(B_{m}+l_{2}\right)}\right] \text {. }
$$

Subtracting (13) from (14) and rearranging terms gives the value of $R$ as

$$
\begin{aligned}
R & =S+S\left[\frac{\left(A_{R}+l_{1}\right)}{\left(B_{m}+l_{2}\right)}-\frac{\left(A_{0}+l_{1}\right)}{\left(B_{m}+l_{2}\right)}\right] \\
& =S\left[1+\frac{\left(A_{R}-A_{0}\right)}{\left(B_{m}+l_{2}\right)}\right] .
\end{aligned}
$$

Since $A_{m}$ and $B_{m}$ equal the respective resistances of the $A$ - and $B$-arms of the DRRS when $A_{m}=B_{m}$, let

$$
\begin{aligned}
& A_{R}=A_{m}+\Delta_{R} \\
& A_{0}=A_{m}+\Delta_{0}
\end{aligned}
$$

where $\Delta_{R}$ and $\Delta_{0}$ are small changes in the resistance of the slide wire. Equation (15) becomes

$$
R=S\left[1+\frac{\left(A_{m}+\Delta_{R}\right)-\left(A_{m}+\Delta_{0}\right)}{B_{m}+l_{2}}\right]
$$

which can be written as

$$
R=S\left[1+\frac{\left(1+\frac{\Delta_{R}}{A_{m}}\right)-\left(1+\frac{\Delta_{0}}{A_{m}}\right)}{\left(1+\frac{l_{2}}{B_{m}}\right)}\right]
$$

since $\frac{A_{m}}{B_{m}}=1$. Now the dials of the DRRS (assumed to be without error) indicate, in effect, that in going from a position corresponding to the resistance $A_{m}$ to that of $A_{R}$, the resistance of the $A$-arm of the DRRS has changed by $\frac{\Delta_{R}}{A_{m}}$ in proportional parts; and similarly, in going from $A_{m}$ to $A_{0}$ the resistance has changed by $\frac{\Delta_{0}}{A_{m}}$. Hence,

$$
\frac{\Delta_{R}}{A_{m}}=\left(D_{R}-D_{m}\right) \text { and } \frac{\Delta_{0}}{A_{m}}=\left(D_{0}-D_{m}\right)
$$

where $D_{R}, D_{0}$ and $D_{m}$ refer to the respective readings of the DRRS translated into proportional parts. Substituting these values in (16) gives

$$
R=S\left[1+\frac{1}{1+\frac{l_{2}}{B_{m}}}\left(D_{R}-D_{0}\right)\right]
$$

or, if $\frac{l_{2}}{B_{m}}$ is sufficiently small 


$$
R \approx S\left[1+\left(D_{R}-D_{0}\right)\right]
$$

In the notation used in developing the general expression given in appendix $\mathrm{B}$, this corresponds to

$$
R_{m, k}=S_{a m}\left[1+\left(D_{m, k}-D_{0}\right)\right] .
$$

It should be noted that the concept of $D_{0}$ has been generalized for the purpose of this paper to indicate that setting on the DRRS for which the ratio arms between terminals 3 and 4 have a $1: 1$ ratio. In most uses of the DRRS, particularly in the comparison of resistance standards, arms $A$ and $B$ contain only the DRRS and $D_{0}$ is a constant, defined as the setting for which the $A$-and $B$-arms of the DRRS have a $1: 1$ ratio.

\section{Appendix B}

Referring to the text and to figure 3 , the working resistance is considered as made up of four groups, with each group containing six sections. The first five sections of any one group have nominally equal resistances and the sixth section has a resistance nominally equal to five times this value (sum of the five sections). Counting from the low-potential end, the first section of each group (after the first) is formed by all the preceding sections connected in series. Any section, therefore, can be identified by two integers; the first, $m$, specifying the group to which the section belongs, and the second, $k$, specifying the position of the section within its group.

If the Wheatstone bridge of figure 4 is balanced as each of the first five sections of group $m$ is inserted in succession in the unknown arm, then the resistance of the first section and of the $k$ th section can be written as,

$$
R_{m, 1}=S_{a m}\left[1+\left(D_{m, 1}-D_{0}\right)\right]
$$

and

$$
R_{m, k}=S_{a m}\left[1+\left(D_{m, k}-D_{0}\right)\right]
$$

where

$S_{a m}$ is the resistance of $S$ and has a nominal value equal to that of the volt box section being measured;

$D_{m, 1}$ and $D_{m, k}$ are the readings of the DRRS in proportional parts ${ }^{7}$ when the first and $k$ th sections, respectively, of group $m$ are inserted in the unknown arm;

$D_{0}$ is the reading that would be indicated on the DRRS if the Wheatstone bridge were in exact $1: 1$ ratio.

Subtracting (19) from (20) gives

$$
R_{m, k}-R_{m, 1}=S_{a m}\left[D_{m, k}-D_{m, 1}\right]
$$

${ }^{7}$ Strictly speaking, as emphasized elsewhere in the paper, $\left(D_{m, 1}-D_{0}\right)$ and
$\left(D_{m, k}-D_{0}\right)$ are the differences in proportional parts as deduced from the readings $\left(D_{m, k}-D_{0}\right)$ are
of the DRRS. or

where

$$
R_{m, k}=R_{m, 1}+S_{a m} d_{m, k}
$$

$$
d_{m, k}=\left[D_{m, k}-D_{m, 1}\right]
$$

The sum of the first five sections of group $m$ is

$$
\sum_{k=1}^{k=5} R_{m, k}=5 R_{m, 1}+S_{a m} \sum_{k=1}^{k=5} d_{m, k}
$$

since each section has the same nominal value and $S_{a m}$ is used throughout the set of measurements.

If now the resistor $S_{a m}$ is replaced by $S_{b m}$ and the bridge is balanced first, with the five sections (connected in series) and then with the sixth section inserted in the unknown arm, we have

$$
\sum_{k=1}^{k=5} R_{m, k}=S_{b m}\left[1+\left(D_{m, s}-D_{0}\right)\right]
$$

and

$$
R_{m, t}=S_{b m}\left[1+\left(D_{m, t}-D_{0}\right)\right]
$$

where

$D_{m, s}$ is the reading of the DRRS when the sum of the five sections is inserted;

$D_{m, t}$ is the reading when the sixth section is inserted.

Subtracting (23) from (24) gives

$$
R_{m, t}-\sum_{k=1}^{k=5} R_{m, k}=S_{b m}\left[D_{m, t}-D_{m, s}\right]
$$

or

where

$$
{ }_{m, t}=\sum_{k=1}^{k=5} R_{m, k}+S_{b m} d_{m, t}
$$

$$
{ }_{m, t}=\left[D_{m, t}-D_{m, s}\right] .
$$

Substituting (22) into (25) gives

$$
R_{m, t}=5 R_{m, 1}+S_{a m} \sum_{k=1}^{k=5} d_{m, k}+S_{b m} d_{m, t}
$$

which defines the resistance of the sixth section of the group $m$ in terms of the sum of the first five sections and the measured differences.

The total resistance of group $m$ is the sum of the resistances of the six sections that comprise the group. Its value is found by adding (22) and (26) so that

$$
R_{m} \equiv \sum_{k=1}^{k=5} R_{m, k}+R_{m, t}=10 R_{m, 1}+2 S_{a m} \sum_{k=1}^{k=5} d_{m, k}+S_{b m} d_{m, t}
$$

Remembering that the total resistance of group $m$ is also the first section of group $(m+1)$ and noting: that

and

$$
S_{a m}=10^{m-1} R_{1,1}
$$

$$
S_{b m}=5 \times 10^{m-1} R_{1,1}
$$


we have from $(27)$

$$
\begin{aligned}
R_{m+1,1}=10 R_{m, 1}+\left(2 \times 10^{m-1}\right. & \left.R_{1,1}\right) \sum_{k=1}^{k=5} d_{m, k} \\
& +\left(5 \times 10^{m-1} R_{1,1}\right) d_{m, t} .
\end{aligned}
$$

Equation (28) serves as the connecting link between one group and the next. If it is applied successively to increasing values of $m$, beginning with $m=1$, one finds that the resistance of the first section of any group $m$ can be expressed in terms of the resistance of the first section of the first group and the measured differences by the general equation

$$
\begin{aligned}
R_{m, 1}=10^{m-1} R_{1,1}+ & \left(2 \times 10^{m-2} R_{1,1}\right) \\
& \quad\left[\sum_{k=1}^{k=5} d_{1, k}+\sum_{k=1}^{k=5} d_{2, k} \ldots+\sum_{k=1}^{k=5} d_{m-1, k}\right] \\
& \quad+\left(5 \times 10^{m-2} R_{1,1}\right)\left[d_{1, t}+d_{2, t} \ldots+d_{m-1, t}\right] .
\end{aligned}
$$

Equation (21) states that the resistance of the $k$ th section of group $m$ is

$$
R_{m, k}=R_{m, 1}+S_{a m} d_{m, k}=R_{m, 1}+\left(10^{m-1} R_{1,1}\right) d_{m, k}
$$

so that the sum of all resistances up to and including the $k$ th section of group $m$ is

$$
\sum_{k^{\prime}=1}^{k^{\prime}=k} R_{m, k^{\prime}} \equiv S_{m, k}=k R_{m, 1}+\left(10^{m-1} R_{1,1}\right) \sum_{k^{\prime}=1}^{k^{\prime}=k} d_{m, k^{\prime}}
$$

Substituting (29) in (30) and factoring out $k \times 10^{m-1}$ $R_{1,1}$ gives

$$
\begin{aligned}
S_{m, k}=k \times 10^{m-1} R_{1,1} & \\
& {\left[1+\frac{\sum_{k=1}^{k=5} d_{1, k}+\sum_{k=1}^{k=5} d_{2, k} \ldots+\sum_{k=1}^{k=5} d_{m-1, k}}{5}\right.} \\
& \left.+\frac{d_{1, t}+d_{2, t} \ldots+d_{m-1, t}}{2}+\frac{\sum_{k^{\prime}=1}^{k^{\prime}=k} d_{m, k^{\prime}}}{k}\right] .
\end{aligned}
$$

Any given ratio of the volt box is defined as the ratio of the sum of all resistances up to the $k$ th tap of group $m$ to the resistance of the first section of the first group. Thus, by definition, any ratio of the volt box is $\frac{S_{m, k}}{R_{1,1}}$ so that, since $\left(k \times 10^{m-1}\right)$ is the nominal ratio, eq (31) can be written in the form

$$
N_{m, k}=\frac{S_{m, k}}{R_{1,1}}=N_{m, k}^{\prime}\left[1+\mu_{m, k}\right]
$$

where $N_{m, k}^{\prime}$ are the nominal ratios and $\mu_{m, k}$ are the corresponding corrections in proportional parts, computed from the difference terms that appear in eq (31). The corrections can be stated in ppm by multiplying by $10^{6}$.

\section{References}

[1] Francis B. Silsbee and Francis J. Gross, Testing and performance of volt boxes, J. Research NBS $\mathbf{2 7}, 269$ (1941) RP1419.

[2] Paul P. B. Brooks, Calibration procedures for directcurrent resistance apparatus, NBS Mono. 39 (1962).

[3] James L. Thomas, Precision resistors and their measurement, NBS Circ. 470 (1948).

[4] Forest K. Harris, Electrical measurements (John Wiley \& Sons, Ine., New York, N.Y., 1952). 